\title{
Effect of surface passivation on generation and recombination lifetimes in silicon wafer studied by impedance spectroscopy
}

\author{
Sanjai Kumar, ${ }^{1}$ P. K. Singh, ${ }^{1, a)}$ and S. R. Dhariwal ${ }^{2}$ \\ ${ }^{1}$ National Physical Laboratory, Dr. K. S. Krishnan Road, New Delhi 110012, India \\ ${ }^{2}$ LM College of Science and Technology, Jodhpur 342001, India
}

(Received 30 December 2009; accepted 18 March 2010; published online 22 April 2010)

\begin{abstract}
Impedance spectroscopy is used to study the effect of surface passivation on minority carrier lifetimes. The technique allows measurement of generation and recombination lifetimes separately. Induced $\mathrm{p}^{+}$-p-n structures are prepared by depositing semitransparent layers of high and low work function metals ( $\mathrm{Pd}$ and $\mathrm{Al}$, respectively) on the two sides of silicon wafers. Hydrogen adsorption property of Pd surface has been utilized for passivation. The generation lifetimes remain almost unaffected but recombination lifetimes enhance many folds after passivations which are in agreement with values obtained by microwave photoconductive decay technique after chemical passivation. Variations are analyzed for estimation of bulk recombination lifetime. (C) 2010 American Institute of Physics. [doi:10.1063/1.3385779]
\end{abstract}

Generation and recombination lifetimes of minority carriers are important for many semiconductor devices. The concept of recombination lifetime $\left(\tau_{\mathrm{r}}\right)$ holds when excess carriers decay as a result of electron-hole recombination. The generation lifetime $\left(\tau_{\mathrm{g}}\right)$ applies when there is a paucity of carriers, as in space charge region of a reverse-biased device while it attains equilibrium. During recombination an electron-hole pair on the average ceases to exist after a time $\tau_{\mathrm{r}}$. When recombination and generation events take place in the bulk, they are characterized by $\tau_{\mathrm{r}}$ and $\tau_{\mathrm{g}}$, and when they occur at the surface, they are characterized by the surface recombination $\left(\mathrm{S}_{\mathrm{r}}\right)$ and generation $\left(\mathrm{S}_{\mathrm{g}}\right)$ velocities. Both the bulk and the surface processes occur simultaneously and the measured lifetime is an effective value $\left(\tau_{\text {eff }}\right)$.

A variety of techniques ${ }^{1,2}$ is available for determination of minority carrier lifetimes in silicon wafers and solar cells. We had recently shown that impedance spectroscopy (IS) can be applied for measurement of both the generation and the recombination lifetimes in silicon. ${ }^{3}$ As the $\tau_{\mathrm{r}}$ value is influenced by $S_{r}$ and sensitive to the surface conditions, surface passivation is important for obtaining the true value of bulk lifetime $\left(\tau_{\mathrm{b}}\right)$. Atomic hydrogen can be used as an effective passivant for both deep and shallow defects in semiconductors ${ }^{4}$ due to its high diffusivity at room temperature. In crystalline silicon, passivation of deep defects is observed in p- and n-type material whereas shallow defect passivation is observed in p-type silicon only. ${ }^{5}$ In this process, hydrogen is dissociated in atomic form ${ }^{6}$ at the metal surface and gets dissolved in the metal. A part of it is adsorbed onto the metal-semiconductor interface, where it gives rise to a dipole layer that changes the metal work function $(\Phi)$ and consequently the electrical properties ${ }^{7}$ of the interface.

In this paper, generation and recombination lifetimes in induced $\mathrm{p}^{+}-\mathrm{p}-\mathrm{n}$ silicon structure are determined by studying the effect of hydrogenation on impedance and $\mathrm{C}-\mathrm{V}$ characteristics. This unambiguously determines the strong passivation dependency of minority carrier lifetimes.

\footnotetext{
a) Author to whom correspondence should be addressed. Electronic mail: pksingh@nplindia.org. Tel.: +91 (011) 45608588.
}

Both side polished p-type $\langle 100\rangle$ silicon wafers (300 $\mu \mathrm{m}$ thick) of different resistivities $(\rho=1000,10$, and $1 \Omega \mathrm{cm}$, referred as $S-1, S-2$, and $S-3$, respectively), are used to make induced $\mathrm{p}^{+}-\mathrm{p}-\mathrm{n}$ structure which is created by depositing semitransparent layers of palladium $\left(\Phi_{\mathrm{Pd}} \sim 5.12 \mathrm{eV}\right)$ and aluminum $\left(\Phi_{\mathrm{Al}} \sim 4.28 \mathrm{eV}\right)$ on the two sides of the wafer at room temperature. The thickness of the Pd film was kept $\sim 300 \AA$ to maintain good optical transparency and that of Al was $\sim 1000 \AA$. No thermal oxide is grown but probability of an ultrathin layer of native oxide at the interfaces cannot be ignored and in such cases measurement sensitivity is rather high. ${ }^{8}$ The details of experimental set-up used in this study are given in Ref. 3.

Figure 1 shows the impedance spectrum in complex plane for sample S-1 at zero bias during the hydrogen adsorption and desorption cycles. The shape of impedance curve is semicircular in vacuum which deviates during hydrogen adsorption process with an appearance of a new but small semicircle at low frequencies. This can be attributed to

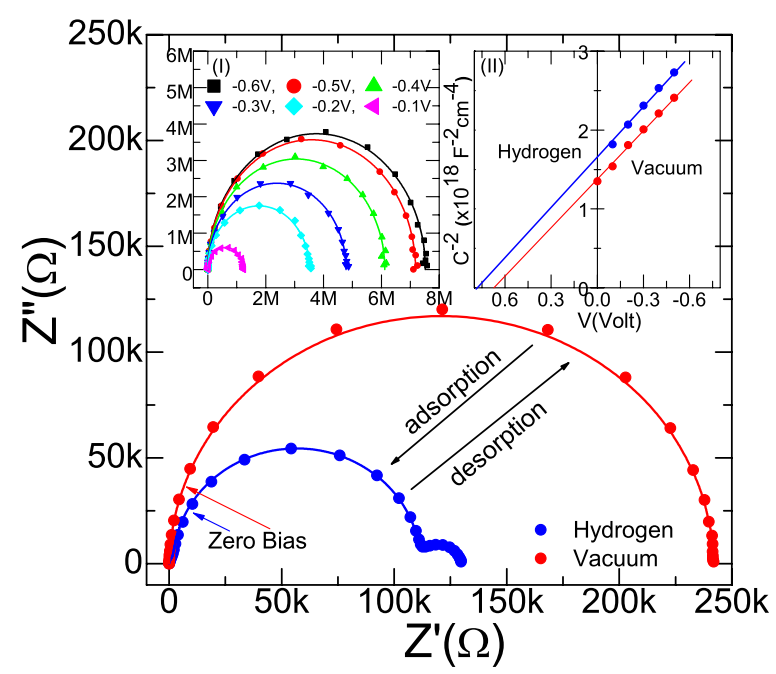

FIG. 1. (Color online) Impedance spectra in hydrogen and vacuum at zero bias for sample $\mathrm{S}-1$. Inset I shows the spectra at different reverse bias $(-0.1$ to $-0.6 \mathrm{~V}$ ) conditions in hydrogen ambient. Inset II represents $\mathrm{C}^{-2}$ vs $\mathrm{V}$ curve in hydrogen and vacuum. The symbols show the experimental points and the lines are the best fit simulated curves. 
TABLE I. The best fit values of $\mathrm{R}, \mathrm{C}$, and $\mathrm{R}_{\mathrm{s}}$ at different $\mathrm{V}_{\mathrm{r}}$ in hydrogen ambient. Values in the brackets show the $( \pm) \%$ error.

\begin{tabular}{cccccc}
\hline \hline $\mathrm{V}_{\mathrm{f}}(\mathrm{V})$ & $\mathrm{R}_{\mathrm{s}}(\Omega)$ & $\mathrm{R}(\mathrm{M} \Omega)$ & $\mathrm{C}(\mu \mathrm{F})$ & $\mathrm{RC}(\mathrm{ms})$ & $\tau_{\mathrm{g}}(\mu \mathrm{s})$ \\
\hline-0.1 & 62.5 & 1.2 & 0.0133 & 16.3 & $\cdots$ \\
-0.2 & 63.3 & 3.5 & 0.0125 & 44.1 & $\cdots$ \\
-0.3 & 63.9 & 4.8 & 0.0119 & 56.6 & $\cdots$ \\
-0.4 & 64.4 & 6.1 & 0.0113 & 69.3 & $\cdots$ \\
$-\mathbf{0 . 5}$ & $\mathbf{6 4 . 7}[\mathbf{3 . 3}]$ & $\mathbf{7 . 8}[\mathbf{5 . 9}]$ & $\mathbf{0 . 0 1 0 9}[3.8]$ & $\mathbf{8 4 . 8}$ & $\mathbf{7 4 . 1}[\mathbf{6 . 4}]$ \\
-0.6 & $65.4[3.2]$ & $7.8[5.7]$ & $0.0103[3.6]$ & 80.6 & $70.4[6.6]$ \\
\hline \hline
\end{tabular}

the hydrogen induced changes in the interface states and bulk acceptor impurities that may affect the effective work function of palladium metal. However, the low frequency side of the spectrum corresponds to surface changes ${ }^{9}$ and hence the observed difference is the manifestation of hydrogen adsorption at the surface. During desorption, impedance spectrum attains its initial shape.

The inset I of the Fig. 1 depicts experimental data with their best fit curves under reverse bias, $\mathrm{V}_{\mathrm{r}},\left(-0.1>\mathrm{V}_{\mathrm{r}}>\right.$ $-0.6 \mathrm{~V}$ ) in hydrogen ambient, where the depletion region edge extends deep into the bulk. The noticeable feature is disappearance of the small semicircle which indicates the reduction in the surface effects. As the nature of data remains semicircular, it can be fitted into a single RC network with one time constant by using the following relation:

$$
Z(\omega)=R_{s}+\frac{R}{1+(\omega R C)^{2}}+j \frac{\omega C R^{2}}{1+(\omega C R)^{2}} .
$$

The best fit $\mathrm{R}, \mathrm{C}$, and series resistance $\left(\mathrm{R}_{\mathrm{s}}\right)$ values for $\mathrm{S}-1$ at different $V_{r}$ are listed in Table I. It is found that the impedance spectrum stabilizes around $\mathrm{V}_{\mathrm{r}}=-0.5 \mathrm{~V}$. The value of $\mathrm{R}_{\mathrm{s}}$ in hydrogen ambient is increased by a factor 2-3 compared to its value in vacuum. ${ }^{3}$ This is in agreement with the results of Laïhem et al. ${ }^{10}$ The $\mathrm{R}$ and $\mathrm{C}$ values are used to calculate $\tau_{\mathrm{g}}\left[=\mathrm{RC}\left(\mathrm{n}_{\mathrm{i}} / \mathrm{N}_{\mathrm{B}}\right)\right]$. The value of base doping, $\mathrm{N}_{\mathrm{B}}$, is calculated from the Mott-Schottky $\left(\mathrm{C}^{-2}-\mathrm{V}\right)$ curves (inset II) and is found $\sim 1.7 \times 10^{13} \mathrm{~cm}^{-3}$ both in vacuum and hydrogen ambient. At $300 \mathrm{~K}, \mathrm{n}_{\mathrm{i}}=1.45 \times 10^{10} \mathrm{~cm}^{-3}$ is considered. ${ }^{3}$ The value of $\mathrm{RC}=84.8 \mathrm{~ms} \quad\left(\right.$ at $\left.\mathrm{V}_{\mathrm{r}}=-0.5 \mathrm{~V}\right)$ gives $\tau_{\mathrm{g}}$ $=74.1 \pm 6.4 \mu \mathrm{s}$ which is close to the value of $\tau_{\mathrm{g}}$ $=73.6 \pm 1.2 \mu \mathrm{s}$ obtained under vacuum $^{3}$ for the same sample. Therefore, the value of generation lifetime is almost the same in vacuum or under hydrogen ambient.

In $\mathrm{p}^{+}$-p-n structure, built-in voltage, $\mathrm{V}_{\mathrm{bi}}$, develops across the two induced junctions of which $\mathrm{Al}-\mathrm{Si}(\mathrm{p}-\mathrm{n})$ contact can accommodate voltage equal to the work function difference of the two materials (i.e., $\Delta \Phi_{\mathrm{pn}}=\Phi_{\mathrm{Al}}-\Phi_{\mathrm{Si}}$ ) and the remaining would appear across $\mathrm{Pd}-\mathrm{Si}$ interface that creates highlow $\left(\mathrm{p}^{+}-\mathrm{p}\right)$ junction. The intercept of $\mathrm{C}^{-2}-\mathrm{V}$ curve on $x$-axis

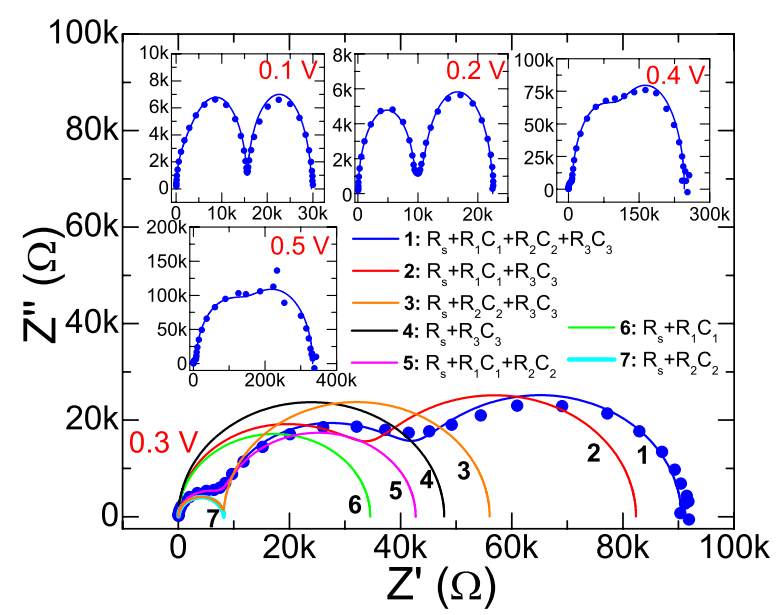

FIG. 2. (Color online) Impedance spectrum under forward bias of $+0.3 \mathrm{~V}$. Insets show the impedance curves for different forward bias in hydrogen. The symbols show the experimental points and the lines are the simulated curves for different RCs and their combinations.

gives the value of $\mathrm{V}_{\mathrm{bi}^{-}} \mathrm{kT} / \mathrm{q}$. The values of $\mathrm{V}_{\mathrm{bi}}$ in vacuum and hydrogen are equal to 0.67 and $0.78 \mathrm{~V}$, respectively (i.e., an increase of $0.11 \mathrm{~V})$. Out of $0.78 \mathrm{~V}\left(\mathrm{~V}_{\mathrm{bi}}\right.$ in hydrogen) Al-Si contact can accommodate only $0.51 \mathrm{~V}\left(=\Delta \Phi_{\mathrm{pn}}\right)$ and the remaining $0.27 \mathrm{~V}$ would appear across $\mathrm{Pd}-\mathrm{Si}$ interface (i.e., high-low junction) as $\mathrm{V}_{\mathrm{hl}}$ (as compared to $0.16 \mathrm{~V}$ in vacuum). An estimate of the $S$ at the edge of the high-low junction can be made by using the relation $S=S_{l} \exp ($ $\left.-q V_{h l} / k T\right)$. The limiting value of surface recombination velocity $\mathrm{S}_{1}$ is equal to $5.2 \times 10^{6} \mathrm{~cm} \mathrm{~s}^{-1}$ at $300 \mathrm{~K}^{3}$ Therefore, increased barrier height in hydrogen effectively reduces $S$ from $\sim 10^{4}$ (in vacuum) to $1.54 \times 10^{2} \mathrm{~cm} \mathrm{~s}^{-1}$ (in hydrogen).

Figure 2 shows the impedance spectrum under forward bias $\left(\mathrm{V}_{\mathrm{f}}\right)$ in hydrogen ambient where $\mathrm{Z}^{\prime}$ versus $\mathrm{Z}^{\prime \prime}$ curves are different from the one obtained under the reverse bias in two ways; (i) they consist of more than one semicircles and (ii) the radii of circles at higher frequency end first decrease up to $+0.2 \mathrm{~V}$ (from its maximum at zero bias) and an opposite trend is observed for $\mathrm{V}_{\mathrm{f}}>+0.2 \mathrm{~V}$. This may be attributed to the increase in barrier potential at the Pd-Si interface after hydrogenation that results in reduction of $\mathrm{S}$ values. A good fitting of the data could be obtained using a more complicated circuit consisting of a number of RC networks connected in series and which is mathematically defined as

$$
Z(\omega)=R_{s}+\sum_{i=1}^{n} \frac{R_{i}}{1+\left(\omega R_{i} C_{i}\right)^{2}}+j \sum_{i=1}^{n} \frac{\omega C_{i} R_{i}^{2}}{1+\left(\omega R_{i} C_{i}\right)^{2}} .
$$

The best fitted $\mathrm{R}$ and $\mathrm{C}$ values with $\mathrm{n}=3$ (e.g., three $\mathrm{RC}$ networks) are given in Table II along with their estimated recombination time constants $\left(\tau_{1}, \tau_{2}\right.$, and $\left.\tau_{3}\right)$, which corre-

TABLE II. The best fit values of $\mathrm{R}_{1}, \mathrm{C}_{1}, \mathrm{R}_{2}, \mathrm{C}_{2}, \mathrm{R}_{3}, \mathrm{C}_{3}$, and $\mathrm{R}_{\mathrm{s}}$ at different forward bias $\left(\mathrm{V}_{\mathrm{f}}\right)$ conditions in hydrogen ambient. $\tau_{1}, \tau_{2}$, and $\tau_{3}$ are the calculated

\begin{tabular}{|c|c|c|c|c|c|c|c|c|c|c|}
\hline $\mathrm{V}_{\mathrm{f}}(\mathrm{V})$ & $\mathrm{R}_{\mathrm{s}}(\Omega)$ & $\mathrm{R}_{1}(\mathrm{~K} \Omega)$ & $\mathrm{C}_{1}(\mu \mathrm{F})$ & $\tau_{1}(\mathrm{~ms})$ & $\mathrm{R}_{2}(\mathrm{~K} \Omega)$ & $\mathrm{C}_{2}(\mathrm{nF})$ & $\tau_{2}(\mu \mathrm{s})$ & $\mathrm{R}_{3}(\mathrm{~K} \Omega)$ & $\mathrm{C}_{3}(\mu \mathrm{F})$ & $\tau_{3}(\mathrm{~ms})$ \\
\hline 0.0 & 52.7 & 19.4 & 16.6 & 322.3 & 1.8 & 15.1 & 27.7 & 17.9 & 0.01 & 0.2 \\
\hline 0.1 & 48.5 & 14.1 & 11.9 & 167.8 & 2.9 & 14.1 & 41.0 & 12.7 & 0.02 & 0.3 \\
\hline 0.3 & 44.9 & 47.8 & 1.3 & 62.1 & 8.2 & 7.4 & 60.5 & 34.5 & 0.07 & 2.5 \\
\hline 0.4 & 44.7 & 138.8 & 0.4 & 59.7 & 7.7 & 7.3 & 19.8 & 99.4 & 0.06 & 6.1 \\
\hline 0.5 & 44.4 & 182.7 & 0.4 & 69.4 & 7.6 & 7.1 & 11.5 & 142.7 & 0.06 & 8.1 \\
\hline
\end{tabular}
lifetimes corresponding to $\mathrm{R}_{1} \mathrm{C}_{1}, \mathrm{R}_{2} \mathrm{C}_{2}$, and $\mathrm{R}_{3} \mathrm{C}_{3}$, respectively. Values in the brackets show the $( \pm) \%$ error in the fitting. 
TABLE III. Generation and recombination lifetime values for S-1, S-2, and S-3 under vacuum (Ref. 5) and hydrogen ambient along with $\tau_{\text {eff }}$ measured (before and after surface passivation) by $\mu$-PCD and $\mathrm{N}_{\mathrm{B}}$.

\begin{tabular}{|c|c|c|c|c|c|c|c|c|}
\hline \multirow[b]{2}{*}{ Sample } & \multirow[b]{2}{*}{$\rho(\Omega \mathrm{cm})$} & \multirow[b]{2}{*}{$\mathrm{N}_{\mathrm{B}}\left(\mathrm{cm}^{-3}\right)$} & \multicolumn{2}{|c|}{ Vacuum (IS) } & \multicolumn{2}{|c|}{ Hydrogen (IS) } & \multicolumn{2}{|c|}{$\mu$-PCD, $\tau_{\text {eff }}$} \\
\hline & & & $\tau_{\mathrm{g}}(\mu \mathrm{s})$ & $\tau_{\mathrm{r}}(\mu \mathrm{s})$ & $\tau_{\mathrm{g}}(\mu \mathrm{s})$ & $\tau_{\mathrm{r}}(\mu \mathrm{s})$ & Unpassivated & Passivated \\
\hline S-1 & 1000 & $1.65 \times 10^{13}$ & 75.3 & 11.4 & 74.1 & 66.1 & 12.09 & 68.50 \\
\hline S-2 & 10 & $2.89 \times 10^{15}$ & 47.4 & 9.9 & 47.9 & 36.2 & 10.26 & 37.51 \\
\hline S-3 & 1 & $4.22 \times 10^{16}$ & 22.3 & 6.5 & 23.6 & 17.3 & 7.14 & 18.86 \\
\hline
\end{tabular}

spond to the two interfaces and the bulk. As an illustration, $\mathrm{Z}^{\prime}$ versus $\mathrm{Z}^{\prime \prime}$ data at $+0.3 \mathrm{~V}$ are plotted in Fig. 2 using $\mathrm{R}_{1} \mathrm{C}_{1}$, $\mathrm{R}_{2} \mathrm{C}_{2}$, and $\mathrm{R}_{3} \mathrm{C}_{3}$ networks individually and their possible combinations. For example, curve 7 obtained by $\mathrm{R}_{2} \mathrm{C}_{2}$ occurs at high frequency end of the spectrum and therefore represents the bulk properties. ${ }^{9}$ The other two $\mathrm{R}_{1} \mathrm{C}_{1}$ and $\mathrm{R}_{3} \mathrm{C}_{3}$ having large $\tau_{1}$ and $\tau_{3}$ could be associated with the metalsemiconductor interfaces on two sides of the device. Curve 1 gives the best fit of the data for the entire spectrum range. The maximum value of $\tau_{2}$ is obtained at $\mathrm{V}_{\mathrm{f}}=+0.2 \mathrm{~V}$ which may be attributed to increased contribution of bulk recombination.

In Fig. 3, simulated curves for $\tau_{\text {eff }}$ as a function of $S$ are plotted for different values of $\tau_{\mathrm{b}}$ by using the expression

$$
\frac{1}{\tau_{\mathrm{eff}, m}}=\frac{1}{\tau_{b}}+\frac{D}{d^{2}} \chi_{m}^{2},
$$

where, $\chi_{\mathrm{m}}$ are the roots of the transcendental equation $\chi_{m} \tan \chi_{m}=S d / D$. Here subscript $m$ represents the $m^{\text {th }}$ root and; $D$ and $d$ are the diffusion constant and thickness of the wafer, respectively. The various modes of Eq. (3) are shown in the inset of Fig. 3 from where it is evident that magnitude associated with higher modes (i.e., $\tau_{\text {eff, }, 2}$ ) is significantly small and could not be detected experimentally. Thus, of the various excitation modes, the fundamental mode $(m=1)$ is indeed the most significant for the interpretation of our data. Figure 3 shows that $\tau_{\text {eff }}$ increases and tend toward $\tau_{\mathrm{b}}$ with the decrease in $\mathrm{S}$. For bulk lifetime $\tau_{\mathrm{b}} \sim 100 \mu \mathrm{s}$, if $\mathrm{S}$ reduces from $\sim 10^{4}$ to $\sim 10^{2} \mathrm{~cm} \mathrm{~s}^{-1}, \tau_{\text {eff }}$ would increase from 10 to $70 \mu$ s. Compared to this, the recombination lifetime value $\left(\tau_{\mathrm{r}}=\tau_{\text {eff }}\right)$ obtained from the best fitted $\mathrm{R}$ and $\mathrm{C}$ values at $+0.2 \mathrm{~V}$ is $\sim 66 \mu$ s (shown in Fig. 3). Thus, we estimate $\tau_{\mathrm{b}}$ $\sim 100 \mu$ s for sample $\mathrm{S}-1$. Further increase in forward bias

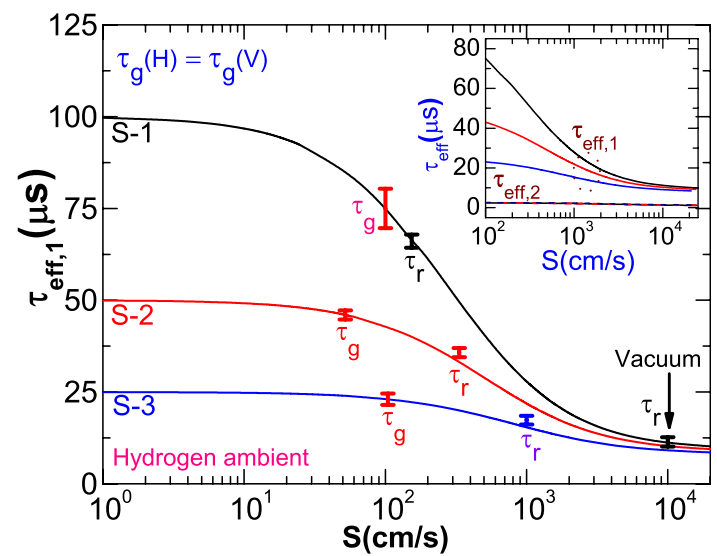

FIG. 3. (Color online) Calculated effective lifetime as a function of surface recombination velocity for different bulk lifetime $\left(\tau_{\mathrm{b}}=25,50\right.$, and $\left.100 \mu \mathrm{s}\right)$. The error bars show lower and upper bounds of the measured $\tau_{\mathrm{g}}$ and $\tau_{\mathrm{r}}$ for S-1, S-2, and S-3 under vacuum and hydrogen ambient. Inset shows the first $\left(\tau_{\mathrm{eff}, 1}\right)$ and the second $\left(\tau_{\mathrm{eff}, 2}\right)$ roots at different $\mathrm{S}$. results in reduction in $\tau_{2}$ as can be seen from Table II. This may be due to voltage drop at the $\mathrm{p}^{+}-\mathrm{p}$ junction as defined by

$$
\Delta V_{\mathrm{hl}}=\frac{k T}{q} \ln \left\{1+\left(\frac{n_{i}}{N_{B}}\right)^{2} \exp \left[\frac{q\left(V-\Delta V_{\mathrm{hl}}\right)}{k T}\right]\right\},
$$

where $\mathrm{S}$ may increase by a factor equal to $\exp \left(\mathrm{q} \Delta \mathrm{V}_{\mathrm{hl}} / \mathrm{kT}\right)$ that results in lowering of $\tau_{\text {eff }}$ or $\tau_{2}$ values.

The results discussed so far are on a high resistivity sample S-1. To verify consistency of our results, measurements were done on low resistivity wafers $(1$ and $10 \Omega \mathrm{cm})$ also. The values of lifetime obtained under reverse $(-0.5 \mathrm{~V})$ and forward bias $(+0.3 \mathrm{~V})$ in hydrogen are shown in Fig. 3. As expected ${ }^{11}$ the low resistivity samples have shorter generation as well as effective recombination lifetimes and hydrogenation increases $\tau_{\text {eff }}$ to the values close to the bulk lifetime of the material as in the case of S-1. For further verification of our experimental results, measurements on the samples of the same batch have also been done by microwave photoconductive decay $(\mu$-PCD) after surface passivation. ${ }^{12}$ All the results are summarized in Table III. A good agreement is found to exist between our results and those obtained from $\mu$-PCD measurements that allows determination of recombination lifetime only.

To conclude, IS can be utilized to study carrier lifetime in silicon by using induced $\mathrm{p}^{+}-\mathrm{p}-\mathrm{n}$ structure and adsorption of hydrogen at the $\mathrm{Pd}-\mathrm{Si}$ interface to passivate the surface in order to reduce surface recombination velocity. Thereby recombination lifetime can be determined and which in hydrogen ambient is found close to the bulk lifetime. However, the generation lifetimes obtained in hydrogen are found close to the values obtained in the vacuum indicating that surface passivation does not affect the generation lifetime.

We acknowledge financial support from CSIR (Grant No. SIP17) and also from MNRE, Government of India as research fellowship to S.K.

${ }^{1}$ S. R. Dhariwal and N. K. Vasu, Solid-State Electron. 24, 915 (1981).

${ }^{2}$ K. Ramspeck, S. Reissenweber, J. Schmidt, K. Bothe, and R. Brendel, Appl. Phys. Lett. 93, 102104 (2008).

${ }^{3}$ S. Kumar, P. K. Singh, G. S. Chilana, and S. R. Dhariwal, Semicond. Sci. Technol. 24, 095001 (2009).

${ }^{4}$ S. J. Pearton, J. W. Corbett, and T. S. Chi, Appl. Phys. A: Mater. Sci. Process. 43, 153 (1987).

${ }^{5}$ R. Rizk, P. de Mierry, D. Ballutaud, and M. Aucouturier, Phys. Rev. B 44, 6141 (1991).

${ }^{6}$ A. Groß and A. Dianat, Phys. Rev. Lett. 98, 206107 (2007).

${ }^{7}$ H. I. Chen and Y. I. Chou, Semicond. Sci, Technol. 18, 104 (2003).

${ }^{8}$ D. Filippini and I. Lundstrom, Appl. Phys. Lett. 82, 3791 (2003).

${ }^{9}$ L. Raniero, E. Fortunato, I. Ferreira, and R. Martins, J. Non-Cryst. Solids 352, 1880 (2006).

${ }^{10}$ K. Laïhem, R. Cherfi, and M. Aoucher, Thin Solid Films 383, 264 (2001).

${ }^{11}$ E. Gaubas and J. Vanhellmont, Appl. Phys. Lett. 89, 142106 (2006).

${ }^{12}$ A. W. Stephens and M. A. Green, Sol. Energy Mater. Sol. Cells 45, 255 (1997) 